\title{
Robust fat saturation applied to late enhancement
}

\author{
Martin A Janich ${ }^{*}$, Jeff A Stainsby ${ }^{2}$, Glenn S Slavin ${ }^{3}$, David Stanley ${ }^{4}$, Christopher J Francois ${ }^{5}$, Scott B Reeder ${ }^{5}$, \\ James F Glockner ${ }^{6}$, Anja C Brau ${ }^{7}$ \\ From 17th Annual SCMR Scientific Sessions \\ New Orleans, LA, USA. 16-19 January 2014
}

\section{Background}

Late Gadolinium Enhancement (LGE) allows imaging of infarction and cardiomyopathies by measuring the accumulation of contrast agent within the myocardium. The shortened $\mathrm{T}_{1}$ relaxation time compared to healthy myocardium is imaged by an inversion recovery (IR) prepared segmented fast gradient echo sequence in which pathology and fat show as bright signal. The fat signal can lead to misinterpretation and poor visualization of epicardial enhancement. In previous work fat was suppressed by using two appropriately timed fat-selective RF pulses which re-invert and invert fat signal [Foo et al., JMRI, 2007] but this technique was sensitive to off-resonance and heart rate variations. The goal of the present work is to make two improvements to fat-saturated LGE:
(1) increase robustness against $B_{0}$ and $B_{1}$ variations by using asymmetric adiabatic RF pulses, and (2) increase robustness against heart rate variations through dynamic timing of fat-selective RF pulses.

\section{Methods}

The selectivity of fat-selective pulses was improved by designing an asymmetric adiabatic inversion pulse based on HS1 and tanh/tan [Hwang et al., JMR, 1999]. This pulse had a transition width of $87 \mathrm{~Hz}$ which is 2 times narrower compared to a symmetric inversion pulse of the same maximum $B_{1}(10 \mu \mathrm{T})$ and pulse duration $(40 \mathrm{~ms}) . \mathrm{M}_{z}$ magnetization depends on the repetition time of the IR pulse and therefore in cardiac gated sequences depends on the patient heart rate. Heart rate variation leads to

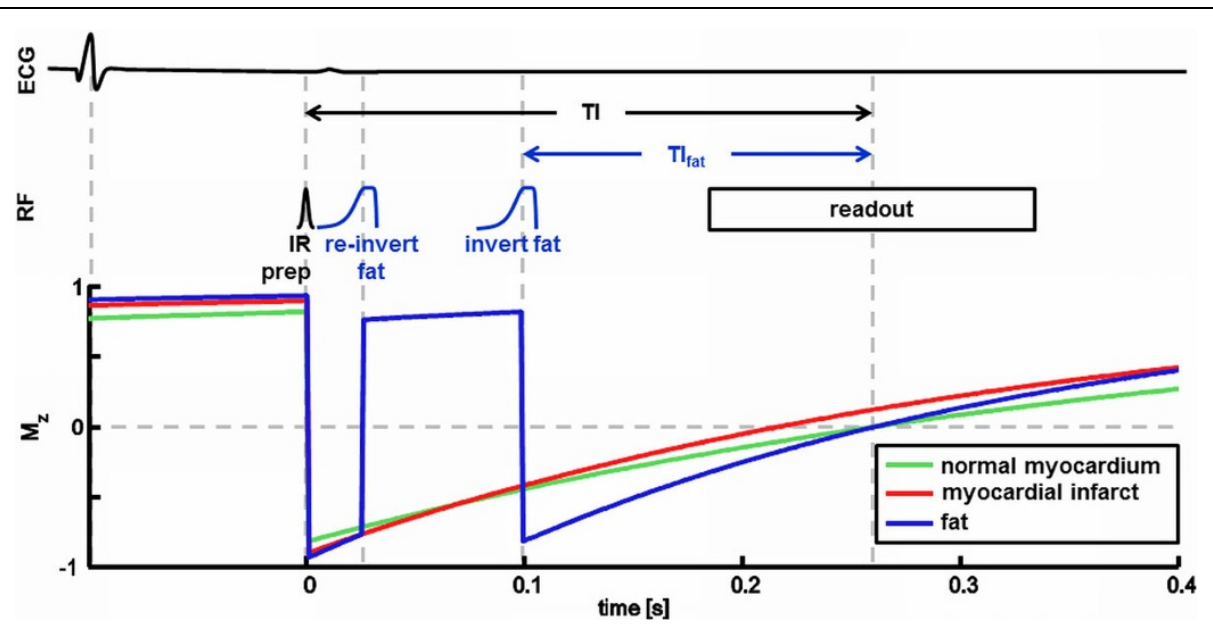

Figure 1 Timing diagram of cardiac gated IR prepared fat saturation pulse sequence (RF phase not shown) and possible $M_{z}$ signal evolution. Signal from normal myocardium is nulled by proper selection of $\mathrm{Tl}$ and signal from fat is nulled by dynamic timing of $\mathrm{Tl}_{\text {fat }}$ which leads to heart rate independent fat suppression.

${ }^{1}$ GE Global Research, Munich, Germany

Full list of author information is available at the end of the article

(C) 2014 Janich et al.; licensee BioMed Central Ltd. This is an Open Access article distributed under the terms of the Creative Commons 

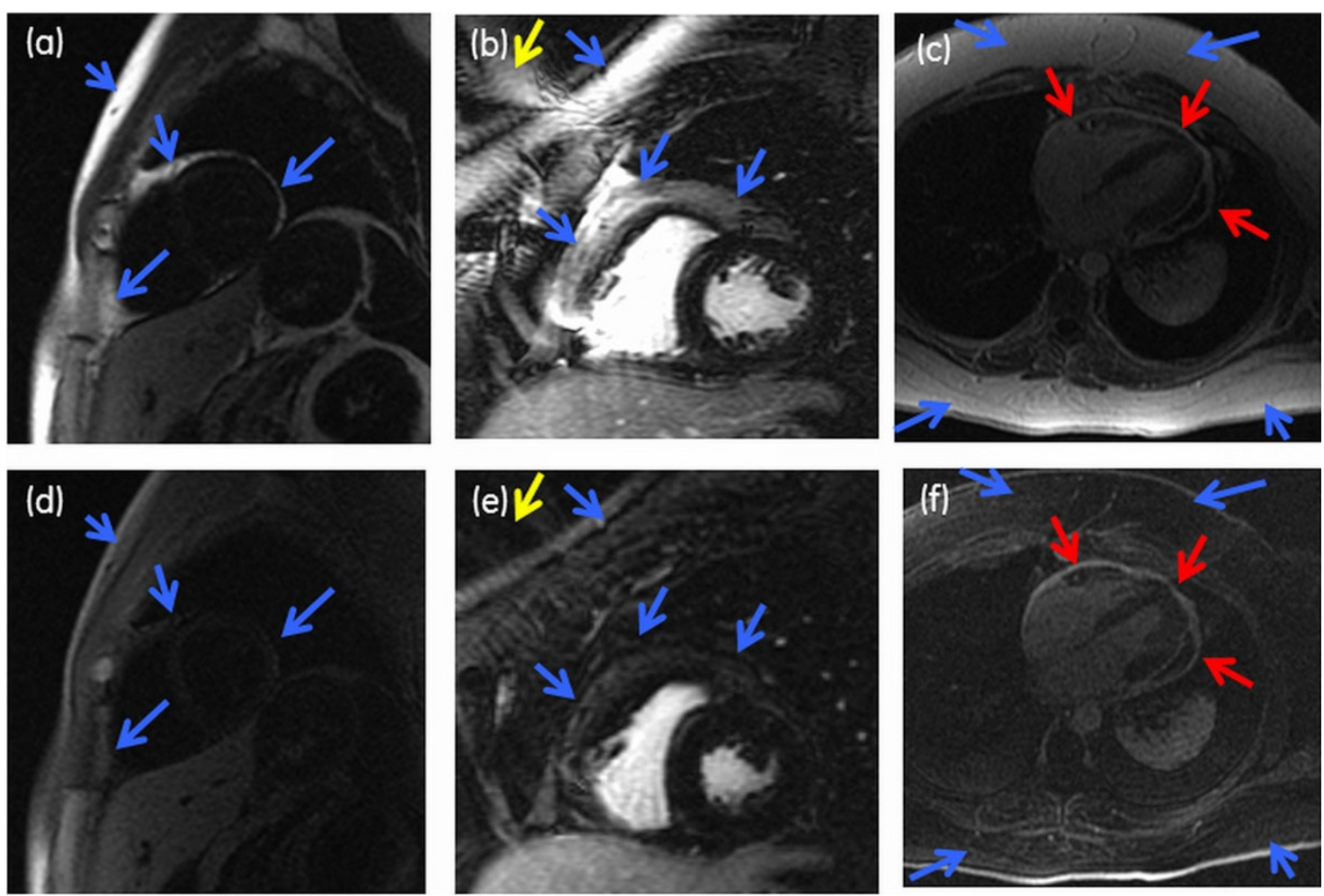

Figure 2 Short-axis IR prepared images from the heart of a $(\mathrm{a}, \mathrm{d})$ healthy volunteer (w/o contrast, $1.5 \mathrm{~T}, \mathrm{HR} \approx 60 \mathrm{bpm}, \mathrm{TI} 280 \mathrm{~ms}$ ), (b, e) patient (w/contrast, 3.0T, HR $\approx 60 \mathrm{bpm}, \mathrm{Tl} 290 \mathrm{~ms}$ ), and (e, f) patient with pericardial enhancement (w/contrast, $1.5 \mathrm{~T}, \mathrm{HR} \approx 80$ bpm, TI 260 ms). The upper row images (a-c) were acquired with IR preparation only and the lower row images (d-f) were acquired with IR preparation and fat saturation. The new technique led to suppression of normal myocardium and fat signal (blue arrows) which avoided image wrapping of subcutaneous fat (e, yellow arrows) and better visualization of pericardial enhancement (f, red arrows).

a shifting of the inversion time $\left(\mathrm{TI}_{\mathrm{fat}}\right)$ at which the fat signal is nulled. Therefore a heart rate independent nulling of fat signal was developed by measuring the length of each $\mathrm{R}-\mathrm{R}$ interval during the examination and time-shifting the fat-selective inversion pulse accordingly (Figure 1). The method was tested in volunteers without contrast and patients with contrast on $1.5 \mathrm{~T}$ (MR450w) and 3.0T (MR750) MR systems (GE Healthcare).

\section{Results}

The $T_{1, \text { fat }}$ value which influences the heart rate-dependent $\mathrm{TI}_{\text {fat }}$ was calibrated in healthy volunteers at field strengths $1.5 \mathrm{~T}(220 \mathrm{~ms})$ and 3.0T (300 $\mathrm{ms})$. With appropriate TI selection the technique led to nulling of signal from healthy myocardium and from fat. The cases without contrast injection had poor blood-myocardium contrast due to the lack of Gd (Figure 2(a)) but fat was successfully suppressed.

\section{Conclusions}

Benefits of fat-saturated LGE imaging include avoiding image wrapping of subcutaneous fat and therefore reduced possible misdiagnosis (Figure 2(b)), and in patients with pericardial enhancement the ability to rule out epicardial fat and better visualization of pathology (Figure 2(c)). The selectivity improvement of fat-selective pulses was particularly important at $1.5 \mathrm{~T}$ where the chemical shift difference between fat and water is only $220 \mathrm{~Hz}$. The technique presented here was proven to be robust in more than 20 cases.

\section{Authors' details}

${ }^{1}$ GE Global Research, Munich, Germany. ${ }^{2}$ GE Healthcare, Toronto, Ontario, Canada. ${ }^{3}$ GE Healthcare, Bethesda, Maryland, USA. ${ }^{4}$ GE Healthcare, Rochester, Minnesota, USA. ${ }^{5}$ Radiology, University of Wisconsin, Madison, Wisconsin, USA. ${ }^{6}$ Mayo Clinic, Rochester, Minnesota, USA. ${ }^{7}$ GE Healthcare, Munich, Germany.

Published: 16 January 2014

doi:10.1186/1532-429X-16-S1-P196

Cite this article as: Janich et al:: Robust fat saturation applied to late enhancement. Journal of Cardiovascular Magnetic Resonance 2014 16(Suppl 1):P196. 